\title{
Dariers disease and pregnancy
}

Julie A. Quinlivan ${ }^{1 *}$ and Louise C. O'Halloran²

*Correspondence: QuinlivanJ@ramsayhealth.com.au

'University of Notre Dame Australia School of Medicine, Centre for Women's and Children's Health,

University of Adelaide, Australia.

${ }^{2} \mathrm{Sir}$ Charles Gairdner Hospital Department of Dermatology.

\begin{abstract}
Darier's disease, also known as keratosis follicularis or Darier-White disease, is an autosomal dominant inherited condition. The disease usually has its onset in the teenage years, meaning it co-exists with the years of fertility in women. The potential dermatological and obstetric implications of Darrier's Disease, especially when it involves the groin and vulva, have not been well reported. We report a case of Darier's Disease associated with multiple antibiotic resistant folliculitis involving skin of the breasts, groin, vulva and perineum that precluded safe vaginal delivery.
\end{abstract}

Keywords: Pregnancy, caesarean section, darier's disease, MRSA, breast feeding

\section{Introduction}

Darier's disease, also known as keratosis follicularis or DarierWhite disease, is a rare autosomal dominant inherited disease with considerable geographical and penetrance variance that affects approximately 1 in 30,000 individuals [1-3]. The condition results from a mutation in the ATP2A2 gene [4]. It affects men and women equally [1]. The disease usually has its onset in the teenage years, meaning it will co-exist with the years of fertility in women [5].

Histologically, Darier's Disease is characterised by dyskeratosis and suprabasal acantholysis, manifesting as warty plaques and papules in seborrheic and flexural regions. It is malodorous and disfiguring; pruritis is common [5]. It often affects the chest, back, neck, forehead, groin and nails [1,2].

The potential implications of Darrier's Disease in pregnancy, especially when it involves the groin and vulva, have not been well reported.

\section{Case report}

A 25-year old woman presented with significant exacerbation of Darier's disease. At 20-weeks pregnancy booking, warty plaques and papules had affected only the seborrheic and flexural regions of the axilla, neck and submammary area. However, there had been disease progression and by 34 weeks, the condition affected both palms, all fingernails, the neck, sub-mammary area, groin and vulva.

In the groin there were significant follicular papules, spreading over the labia minor, labia majora, perineum and perianal area. There was gross fissuring of the skin and leakage of haemoserous fluid (Figure 1).

In addition, papules had thickened the perineal skin; the tissue was inelastic and rubbery with a superimposed inflammatory component (Figure 2).

There was widespread evidence of infected follicles, including the area where a Pfannenstiel incision would be performed should a caesarean section be required, as well as the perineal and forchette region where the fetal head would crown in a vaginal delivery.

On obstetric examination, the fetus was normally grown, cephalic presentation, with normal fetal heart rate. An obstetric ultrasound examination and cardiotocographic trace were normal.

Swabs were collected from the sub-mammary, groin, vulval and vaginal area. Cultures demonstrated a heavy growth of Methicillin resistant Staphylococcus Aureus (MRSA) and a multiple antibiotic resistant Group B Streptococcus. Viral culture was negative, including culture for herpes virus. Treatment was complicated by the patient's known allergy to penicillin. Hospital admission was arranged at 36 weeks for infection review, skin optimisation and delivery planning. Bandages soaked in chlorhexidine obstetric lotion were applied to the lower abdomen across the site of potential Pfannensteil incision. In addition, gauze swabs soaked in Mometasone Furoate ointment were applied to the perineum and Mometasone Furoate cream applied to the remaining vulval area.

The skin of the lower abdomen cleared considerably after 48 hours. However, the vulval skin remained thickened, fissured and continued to leaked haemoserous fluid. There was little elasticity in the vulval skin precipitating concern that the vulva would shatter with crowning of the fetal head. After discussion, caesarean section delivery was booked.

Following clinical microbiology review, it was decided not to treat the ongoing infections with antibiotics and instead to use anti- staphylococcal washes for two days before delivery and to prophylactically cover delivery with Vancomycin for 48 hours. Due to the risk of newborn Group B Streptococcus infection, the baby was to be administered prophylactic penicillin from birth.

The patient was admitted at term and delivered by caesarean 


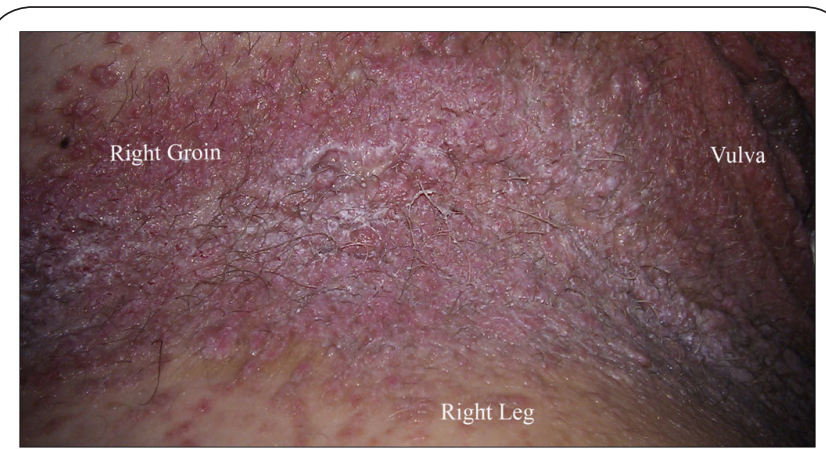

Figure 1. Right groin region showing hyperkeratotic, inelastic skin with superimposed inflammatory component and significant follicular papules present.

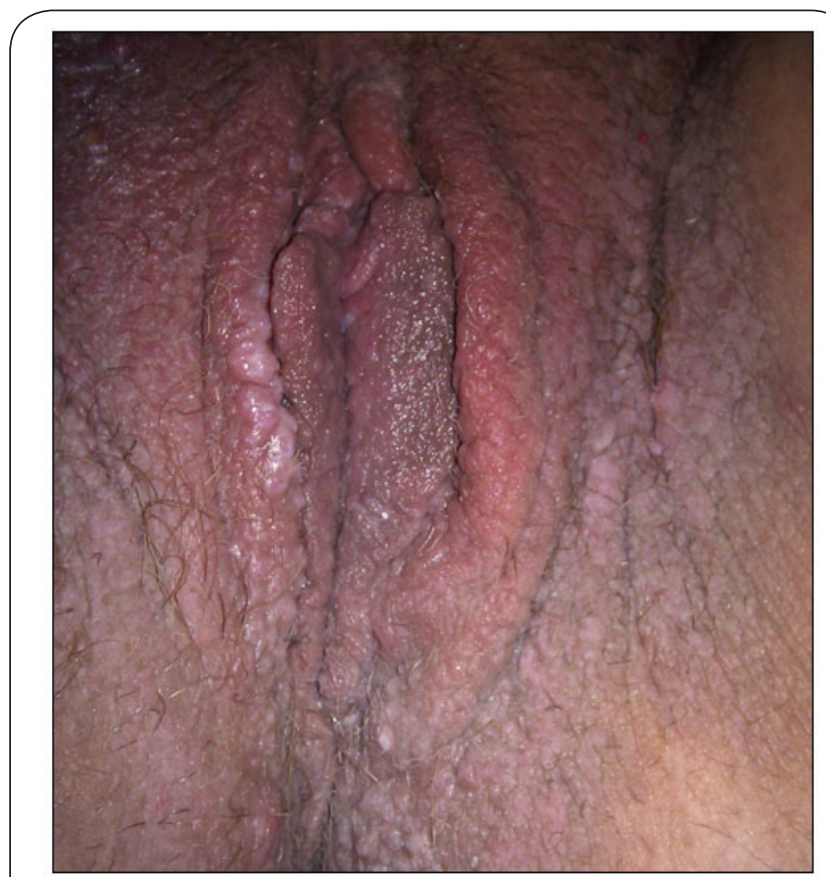

Figure 2. Vulval region also showing hyperkeratotic inelastic skin with superimposed inflammatory component with follicular papules.

section. There were no post-operative complications.

At six-week review, the wound was healed. However, there was further exacerbation of disease affecting the breasts. Although there was nipple sparing, the inferior half of each breast and the area from the sub-mammary region to the umbilicus was now affected. The mother reported pain when holding the baby against the breast and had decided to formula feed. On formal screening there was no symptomatology consistent with postnatal depression.

\section{Discussion}

This is one of the few case reports of pregnancy complicated by Darier's disease. In this case exacerbation of disease resulted in caesarean section delivery and early cessation of breastfeeding due to involvement of the groin, vulval, forchette and breast skin. There have been limited publications on Darier's disease in pregnancy [6-9].

Darier's disease is an obstetric issue because the autosomal dominant pattern inheritance means that $50 \%$ of offspring will be affected. As a result, couples should be offered genetic counselling at a preconception visit or offered referral to a high-risk obstetrician early in the pregnancy for counselling. Prenatal diagnosis has been possible since the 1980s [10].

Prenatal counselling is difficult in Darier's disease due to variable penetrance of disease; it is not possible to predict the severity of disease in the offspring of affected individuals and genotype-phenotype correlation studies have been disappointing [4]. Darier's disease carries a wide spectrum of disease phenotypes, with many mild forms of the disease remaining undiagnosed and severe forms carrying considerable quality of life impacts.

The varying phenotypes mean that counselling is important, as women with mild phenotypes may give birth to children with severe phenotypes. Lack of understanding of genetic expression may lead to considerable anger, anxiety and guilt in parents if the child subsequently develops a severe phenotype of the disease and the couple feel they were not adequately prepared for this outcome. One mother whose son had a severe phenotype is reported in the literature as stating, "I would never have had children if I had known it could do this." [5].

Severe disease is usually treated with oral and/or topical retinoids. These therapies elicit a positive clinical response in $90 \%$ of patients. However, they are teratogenic and contraindicated in pregnancy. Topical steroids may be safely used in pregnancy but have poor efficacy $[2,5]$.

As demonstrated in the present case study, Darier's disease may cause pregnancy complications when there is skin involvement of the groin, vulva or perineum where skin elasticity is important for atraumatic vaginal birth, or when it involves the lower abdomen where Pfannensteil incision for caesarean section delivery may be required. It may also affect delivery if there is widespread back involvement that precludes safe administration of regional anaesthesia [7]. Histologically in Darier's disease there is acantholysis that results in suprabasal clefting with papillomatosis and dyskeratosis $[6,11]$. Electron microscopy shows loss of desomosomal protein attachments and perinuclear aggregation of keratin filaments [11]. It has been suggested that the primary target of the chromosomal mutation is the desmosomal plaque [11]. The histological skin changes predispose to infection through barrier thinning.

The presence of superimposed infections causes obstetric problems, especially in respect to Group B Streptococus that can lead to specific neonatal sepsis and death. Allergy to Penicillin and multiple antibiotic resistance compromised antibiotic management further in this case. The development 
Quinlivan et al. Dermatology Aspects 2013,

of antibiotic resistance may occur in the setting of recurrent infections where bacterial colonisation of the skin is difficult to eradicate.

Skin infections may also affect breastfeeding if the breast is involved. In the present case localised fissuring resulted in breast pain that lead to discontinuation of breastfeeding. Neuropsychiatric associations have been reported in Darier's disease. The reported associations are predominantly mood disorders, which affect $50 \%$ of patients. The common expressions of these disorders are depression with suicidal ideation and suicide attempts [12]. In the context of pregnancy, this pre-existing vulnerability predisposes the mother to postnatal depression. It is important to formally screen for depression at postnatal review.

We present a case of Darier's disease in pregnancy and focus on the need for prenatal counselling, review of delivery modality and vulval skin elasticity, and the need to provide input for breastfeeding and postnatal depression screening.

\section{Competing interests}

The authors declare that they have no competing interests.

\section{Authors' contributions}

J.A.Q and L.C.O were involved in drafting the manuscript for publication. JQ was responsible for overall clinical care of the patient and LO'H was responsible for dermatology input.

\section{Acknowledgement}

We acknowledge the patient who provided consent to have her case reported.

\section{Publication history}

Received: 28-Jun-2013 Revised: 18-Aug-2013

Accepted: 20-Aug-2013 Published: 28-Aug-2013

\section{References}

1. Synopsis of Clinical Dermatology - ${ }^{\text {th }}$ Ed. New York. The McGraw-Hill Companies.

2. Cooper SM and Burge SM. Darier's disease: epidemiology, pathophysiology, and management. Am J Clin Dermatol. 2003; 4:97-105. I PubMed

3. Munro CS. The phenotype of Darier's disease: penetrance and expressivity in adults and children. Br J Dermatol. 1992; 127:126-30. | PubMed

4. Sakuntabhai A, Burge S, Monk S and Hovnanian A. Spectrum of novel ATP2A2 mutations in patients with Darier's disease. Hum Mol Genet. 1999; 8:1611-9. | Article | PubMed

5. Hulatt $L$ and Burge $S$. Darier's disease: hopes and challenges. J $R$ Soc Med. 2003; 96:439-41. | Article | PubMed Abstract | PubMed Full Text

6. Carrillo Dde L. Vegetating darier's disease during pregnancy. Acta Derm Venereol. 2006; 86:259-60. | Article | PubMed

7. Sharma R, Singh BP and Das SN. Anesthetic management of cesarean section in a parturient with Darier's disease. Acta Anaesthesiol Taiwan. 2010; 48:158-9. | Article | PubMed

8. Espy PD, Stone $\mathrm{S}$ and Jolly HW, Jr. Hormonal dependency in Darier disease. Cutis. 1976; 17:315-20. | PubMed

9. Spouge JD, Trott JR and Chesko G. Darier-White's disease: a cause of white lesions of the mucosa. Report of four cases. Oral Surg Oral Med Oral Pathol. 1966; 21:441-57. | Article I PubMed

10. Shimizu $\mathrm{H}$ and Suzumori K. Prenatal diagnosis as a test for genodermatoses: its past, present and future. J Dermatol Sci. 1999;
19:1-8. | Article | PubMed

11. Hakuno M, Shimizu H, Akiyama M, Amagai M, Wahl JK, Wheelock MJ and Nishikawa T. Dissociation of intra- and extracellular domains of desmosomal cadherins and E-cadherin in Hailey-Hailey disease and Darier's disease. Br J Dermatol. 2000; 142:702-11. | Article | PubMed

12. Gordon-Smith K, Jones LA, Burge SM, Munro CS, Tavadia S and Craddock $\mathrm{N}$. The neuropsychiatric phenotype in Darier disease. Br J Dermatol. 2010; 163:515-22. | Article | PubMed

\section{Citation:}

Quinlivan JA and O'Halloran LC. Dariers disease and pregnancy. Dermatol Aspects. 2013; 1:1. http://dx.doi.org/10.7243/2053-5309-1-1 